\title{
Discuss of Key Issue for Automatic BATS Control in Regional Power Grid
}

\author{
Kaiyan Pan, Yingying Sun, Zhenyan Gao \\ Dongfang Electronics Corporation, Yantai, China \\ Email: ytdfsunyy@163.com
}

How to cite this paper: Pan, K.Y., Sun, Y.Y. and Gao, Z.Y. (2017) Discuss of Key Issue for Automatic BATS Control in Regional Power Grid. Energy and Power Engineering, 9, 189-197. https://doi.org/10.4236/epe.2017.94B023

Received: January 22, 2017

Accepted: March 30, 2017

Published: April 6, 2017

\begin{abstract}
Busbar Automatic Transfer Switch (BATS) is very important for power distribution reliability. However, BATS can't consider whether it cause overloading of devices after it acts. In this paper, we introduce the design principle of Automatic BATS control from whole architecture including its function, strategy and a rule of on-off. On the other hand, the running experience and effect is also introduced. Site operation shows that the proposed method is feasible and it can ensure power grid reliability.
\end{abstract}

\section{Keywords}

Busbar Automatic Transfer Switch (BATS), N Minus One, On-Line Controlling, Automaitc BATS Control

\section{Introduction}

Usually, the main transformers and lines of regional power grid are under $220 \mathrm{kv}$ voltage level. Some $500 \mathrm{kv}$ main transformers are also included in the regional power grid. These $220 \mathrm{kv}$ lines make a ring network, while the $110 \mathrm{kv}$ devices run in radial power network. To ensure the reliability of power supply, a few of busbar automatic transfer switches (BATS) are used and installed in substation. When the power grid has fault and the power supply has been broken, these BATS work to recover the power supply [1].

Smart grid firstly is strong power grid including of extra-high voltage network as the main grid and several coordinated networks with different voltage levels. In addition, the flexibly used of advanced communication technology, information technology and control technology make the smart grid informational, automatical and interactional. The smart grid has several basic characteristics such as self-healing, integration, interaction, optimization, compatible and so on. Selfhealing is based on real time measurement and forecast of power grid operating 
data to achieve the detection, rapid diagnosis and recovery processing of power grid faults. When a power grid fault occurs, the self-healing can isolate the fault and handle the fault quickly with no or less manual intervention to avoid the power cut over a large area [2].

The self-healing devices can be installed in the substation or the power supply center. A method of cutting load automatically was provided to deal with the over load operating of power equipment [3]. An automatic load transfer control technology was advanced to deal with the over load operating of $330 \mathrm{kv}$ main transformers [4]. To assure the accuracy and validity of load-cutting, a cooperative load-cutting control method of intelligent transformers was supplied [5]. Load transmission can also be used to remove the over load problem of high voltage level power devices [6]. All these methods forenamed only can be used for dealing with power devices over load while the power supply is normal. However, when the devices have fault and the BATS has acted which causes new device over load, these methods are invalid. For this reason, it is important to analyze the operation state of power networks intensively after the BATS acting, and the acting BATS will be locked beforehand if new over load is consider to will happen again. An automatic on/off control method of BATS press plate is provided in this paper, which considers of the oil temperature of main transformer, initial load factor of main transformer and the N-1 check.

\section{Overall Framework of Automatic BATS Control System}

Automatic BATS control system (ABC) is developed based on Energy Management System (EMS). The ABC system can get the real-time measurement data from Supervisory Control and Data Acquisition (SCADA) as the basic data and considers of several impact factors such as the oil temperature of main transformer, initial load factor of main transformer, the sequential parameters of $\mathrm{N}-1$ check, priority level of BATS, data exception and so on. The ABC system will calculate and output the BATS control plan online. The ABC system can control the BATS press plate real time and online and achieve the automatic on/off of BATS by remote control technology of secondary equipment. The overall framework is shown in Figure 1.

As shown in Figure 1, the ABC system is running based on SCADA/EMS can provide the control plan of BATS. Firstly, the $A B C$ system gets the real time on/off state of BATS by SCADA. Secondly, the ABC system runs the data detection function and power system state estimation function and then makes the BATS control plan which has considered of the oil temperature, initial load factor of main transformer and N-1 check. Finally, the control plan will be handed down to the BATS press plate to control the BATS on/off.

The $\mathrm{ABC}$ system can supply guard signals if there are remote operation errors.

\section{Functions of Automatic BATS Control System}

\subsection{Set of Global Control Parameters}

Control parameters needed to be set include control rules, check rules and global 


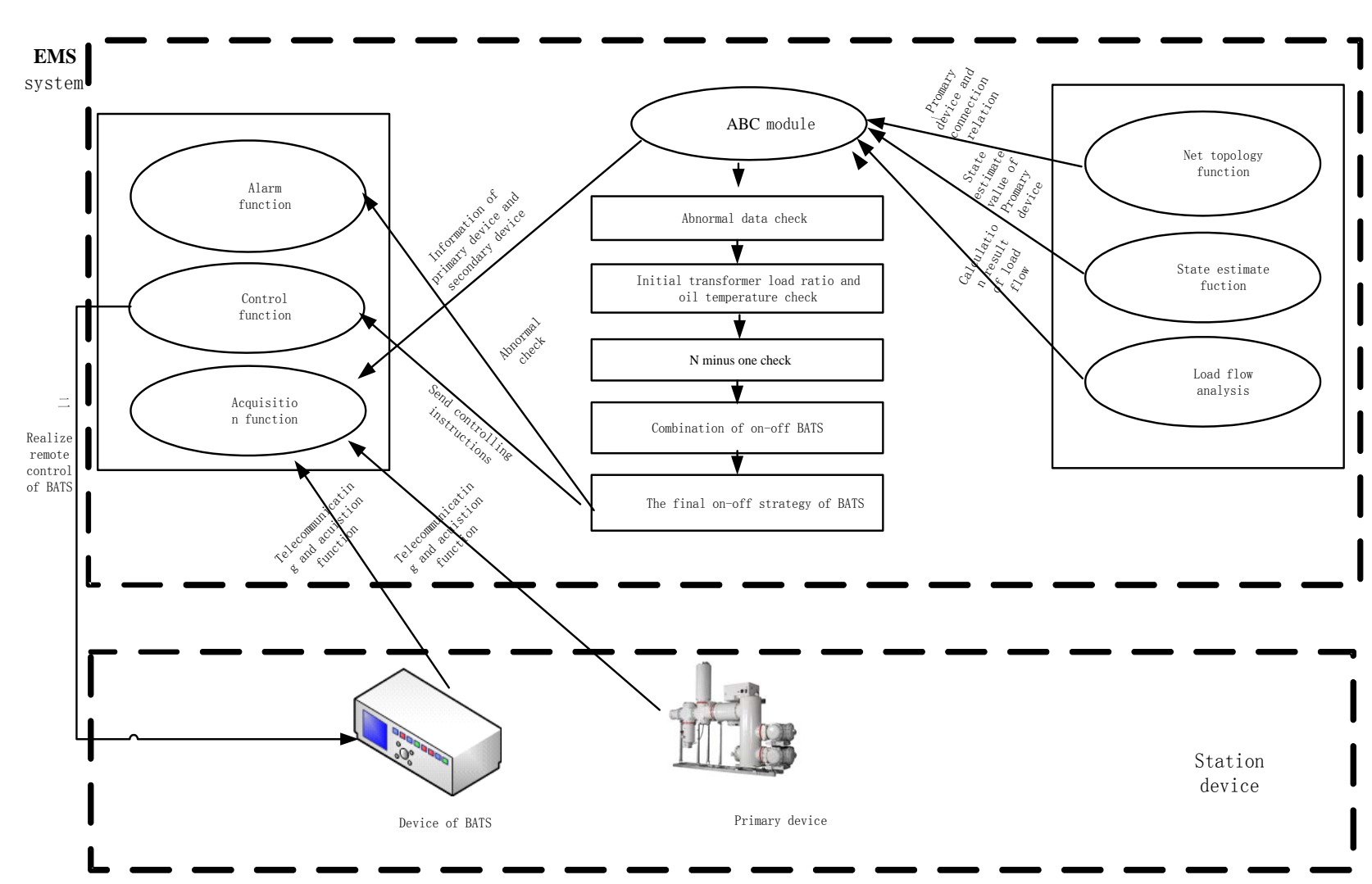

Figure 1. The whole architecture of $\mathrm{ABC}$.

control parameters.

1) Control rules

N-1 Rule: selection of several calculations such as N-1 calculation of $110 \mathrm{KV}$ main transformer, N-1 calculation of $110 \mathrm{KV}$ lines, N-1 calculation of $220 \mathrm{KV}$ main transformer and so on.

Other Rules: such as selection of check calculation of oil temperature, selection of check calculation of initial load factor of main transformer and so on.

2) Check rules

Check rules: selection of several check calculations such as over load check calculation of $110 \mathrm{KV}$ main transformer, over load check calculation of $110 \mathrm{KV}$ lines, over load check calculation of $220 \mathrm{KV}$ main transformer and so on.

3) global control parameters

a) Running selection of BATS;

b) Operation cycle of calculation, default is 10 minutes;

c) Limit value of single BATS action number, default is 15 for one day.

\subsection{Overall Check Rule}

The ABC system considers of several impact factors such as the oil temperature, initial load factor of main transformer and N-1 check and so on. Generally speaking, if the oil temperature check is failed, the $\mathrm{ABC}$ system quit and if the oil temperature check is successful, then the ABC system runs the N-1 check calculation. 


\section{Key Issue for Automatic BATS Control System}

\subsection{Model of Automatic BATS Control System}

An automatic devices allocation plan of Energy Management System/Dispatcher Training Simulator (EMS/DTS) is provided [7]. BATS factory built the BATS model according to the characteristic of their database. The BATS model in the ABC system can be looked in scientific paper [8]. This BATS model can provide the general design sufficiently considering of functions of EMS/DTS and BATS press plate. This model includes BATS type (coupler BATS or device BATS), BATS devices (line or transformer), switches, bus and so on. This common model of BATS is shown in Figure 2, in which hollow switch means open and solid switch means close. For ABC modules, there are some fields needed to be extended, such as on/off set (local quit, local put into, automatic control), on/off adjust mode (stop, start, closed loop), press plate signal location, split signal location, charge signal location and so on.

\subsection{Optimum Control Policy of BATS Press Plate Considering of Main Transformer Oil Temperature and N-1 Check}

\subsubsection{Control Policy of BATS Press Plate Considering of Main Transformer 0il Temperature}

The over load ability in short time of transformer is distinctly impacted by its oil temperature, and the oil temperature can adequately mirror the current operation state of transformer. Therefore, transformer oil temperature is considered as a control parameter of BATS control system. For a transformer located with BATS device, when its oil temperature over a set value, this BATS will be off. On the other hand, this BATS may be need to be on.

For the control policy of BATS press plate considering of main transformer oil temperature and initial load factor, it is necessary to do check calculation of both $220 \mathrm{kv}$ and $110 \mathrm{kv}$ main transformer oil and initial load factor.

\subsection{2. $\mathrm{N}-1$ Check}

A new N-1 fault analysis method is advanced for which is the causation of BATS action according to the characteristics of regional power grid [9]. This method is based on original topology analysis, and uses the method of key supply routings searching to make the conforming BATS to act when there are devices faults. The power supply location is the start node which generally is $220 \mathrm{kv}$ bus or

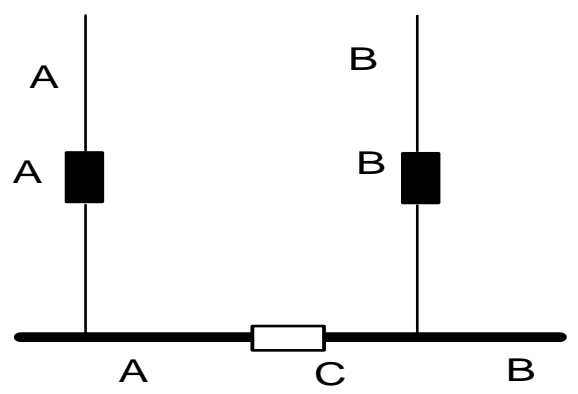

Figure 2. Common model of BATS. 
modeled generation, and the searching routing is up to down and will be recorded. The load transfer is used for check calculation. Because of topology calculation is not need to be repeated again, the calculated speed can be improved obviously and the calculation time can be reduced.

\subsection{Control Policy of BATS Based on Multiple Objective Optimization}

Suppose that some specific N-1 fault will cause BATS with number $\mathrm{n}$ to act, several factors such as the load value, the current BATS state and so on should be considered in the actual execution control policy. Moreover, the number of off-line BATS should be as lower as possible. So, if some BATS will act in several $\mathrm{N}-1$ faults, then this BATS should be prior to be off line in the final BATS control plan.

As above, there are several conditions should be considered in the off-line control policy. First, the regional state of BATS, for example, on line is 0 and off line is 1 . Second, the transferred load value by BATS, generally speaking, the smaller load has high off-line priority and keep the BATS with large load on line as much as possible. Third, the numbers and locations of BATS shown in different $\mathrm{N}-1$ faults, the bigger the number, the higher priority the BATS off line. In actual processing and control, the priority of different BATS devices can be set different priorities according to the request of users and final priority of BATS can be obtained after synthesis.

\subsection{Bad Data Processing}

The on/off control policy is based on main transformer oil temperature check calculation and real time $\mathrm{N}-1$ check calculation, there are some kind of possible bad data should be noticed.

- The anomaly main transformer oil temperature data of correlated transformer. If the oil temperature is considered in BATS control, the current operation state of the BATS press plate keeps constant.

- If there are bad data about $220 \mathrm{kv}$ main transformers located BATS detected in state estimation calculation, the correspondent $220 \mathrm{kv}$ main transformer check calculation will be skipped.

- If there are bad data about $110 \mathrm{kv}$ main transformers or lines located BATS detected in state estimation calculation, the current operation state of the BATS press plate keeps constant.

\subsection{Concurrent Remote Control Processing}

The on/off control policy of BATS ordinarily is executed once every 10 minutes. In the peak period of load, there ordinarily have hundreds of BATS needed to executed on/off control. Mostly, these BATS belong to different substations and the remote control time of single press plate is 5 seconds, if the remote control processing is sequential, then the total remote control execution time estimates to be 20 minutes. Obviously, this time cannot satisfy the request of real-time. For 
this reason, the concurrent remote control processing is necessary to be used. With concurrent remote control processing, these BATS of different substations can be remote controlled in the same time and the total remote control time is equal to the maximum remote control time of all these substations. So, the remote control time can be reduced obviously.

\subsection{Abnormal Condition Processing}

Transformer oil temperature check and N-1 check (including $110 \mathrm{kv}$ lines N-1 check) are the major conditions for the $\mathrm{ABC}$ system policy calculation. N-1 check calculation is based on the state estimation calculation and power flow calculation. In some special case, the power flow calculation cannot be convergent. If the power flow misconvergence occurs continuously for several cycles, correspondent abnormal condition processing should be started. The abnormal condition processing is according to the history policies. For example, if some BATS have been off line in the past week, then in this case this BATS will be off line again. At the other hand, the BATS will be on line if it was on line in the past week.

There are also some other abnormal conditions needed to be noticed, such as non-refresh of SCADA telemetering data, misconvergence of state estimation calculation, abnormal data of maximum number of BATS remote controlled every hour, abnormal data of maximum number of BATS remote controlled every day and so on.

\subsection{Time Characters and Equipartition Characters}

\subsubsection{Time Characters}

When the power grid has faults, the power supply of lots of bus may be cut. The higher voltage level that fault device has, the more buses with power supply cut. If there is no interoperation in multiple BATS, the number of executed BATS will increase a lot and in fact no all the BATS execution is necessary. Therefore, the time characters of BATS on/off control must be noticed in the BATS press plate control policy.

\subsubsection{Equipartition Characters}

After the action of some BATS, the load is uniformly distributed to these standby power to avoid the over load of some standby power causing the action of correspondent BATS, which is defined as the equipartition characters of BATS. As shown in Figure 3, suppose the transformer A had wrong and the equipartition is ignored, all the load of bus A, B1, B2 will transfer to transformer B. But if the equipartition is considered, the BATS of transformer B and transformer $\mathrm{C}$ will both, then the load of bus A and B1 will be distributed to transformer B and the load of bus B2 and C will be distributed to transformer C. Obviously, by the equipartition characters, the possibility of transformer B over load will be reduced.

A detail process method is discussed about the time characters and equipartition characters [10]. Generally speaking, for the sequential processing, if the 


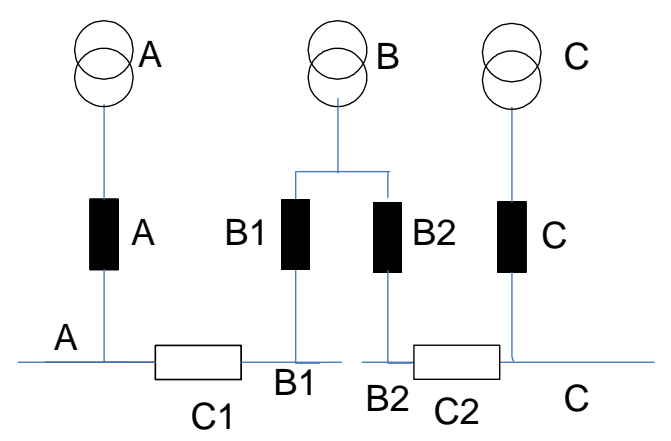

Figure 3. Split-characteristics of BATS.

BATS with higher voltage level has acted, then these BATS with lower voltage level will be ignored. And the equipartition of BATS can be simulate by resolving BATS into master BATS and slave BATS.

\subsection{Hierarchical Check Processing}

The target of the $\mathrm{ABC}$ system is assuring the reliability of power grid supply by real time BATS on/off control and avoiding the over load of devices after BATS action when the power grid has wrong. For some reasons, it is no possible that all the BATS act in closed loop. However, the policy message of these BATS also is needed to submitted to the dispatcher. It requires that the $\mathrm{ABC}$ system not only provide on/off control plan and remote control command of these BATS operating in closed loop, but also the control plan of other BATS not operating in closed loop. To avoid the interaction of BATS control policy with different control modes, hierarchical check calculation is necessary. The check calculation and the control policy decision of BATS operating in close loop can be executed firstly, and then turn to the BATS not operating in close loop.

\subsection{The ABC System Test Technology}

For most cases, rigorous test in open loop must be executed before the BATS is put into operation in close loop. There are several test items, as follows.

- Testing about if the signals of BATS press plate has been correctly acquired or not.

- Testing about if the policy flag in database is set in open loop or not.

- Testing about these relevant parameters of graphics primitives in BATS information pattern is correct or not.

- Testing about if the load factor and N-1 check are included in the global control parameters or not.

- Testing and making sure that all the switches places except the BATS press plate signal which can be controlled in the substation are on the spot.

- Testing about the BATS press plate signals.

- Setting the current of transformer manually, for the three winding transformer its high voltage current is chosen. And make sure that the transformer load factor is 0.85 . Testing that if the policy is correct or not. 
If all the above tests have been passed, the correspondent BATS can be put from open loop into closed loop.

\section{Application Analysis}

The BATS are very important to assure the reliability of power supply. The ABC system discussed in this paper has been used in GuangZhou power grid. The influence of primary network and load characters is obvious for GuangZhou power grid. In the peak period of load especial in summer, the BATS action of some heavy load substation causes the over load of other devices. For the conventional BATS on/off management, lots of BATS will be off line for a long time.

\subsection{The ABC System Application and Efficiency}

Before the $\mathrm{ABC}$ system is used, system operation apartment of GuangZhou power grid needs to assign lots staffs to calculate and analyze the BATS press plate $0 \mathrm{n} /$ off control plan of overall the grid which is almost one day with one person every week. And the on/off line of BATS is need the staff to finish manually. The ABC system can help the GuangZhou power grid to reduce the human consumption about 112 hours of one person in every season, and improve the working efficiency. Also, the ABC system can solve several problems such as the calculation real-time performance and the rapidity of BATS on/off line. The $\mathrm{ABC}$ can raise the accuracy of BATS control actions.

The ABC system has been put into close loop operation in 2014 Jun. The average percentage of BATS on-line for heavy load substation has raised over $60 \%$. The average number of remote control in one day is over 100 and the success percentage of remote control is $100 \%$. The $\mathrm{ABC}$ system realizes the detailed management of BATS operation, reduces the stress of dispatcher from urgent power recover, and improve the working efficiency.

\subsection{Application Example}

At 21:17 p.m. of 28th Jan 2015, the HuangHua $110 \mathrm{kv}$ line of HuangPu power plant got lasting broke. The \#2 main transformer of NanGang substation, the \#2 main transformer of YunPU substation and the \#2 main transformer of HeCun substation all lost voltage. In this case, by the ABC system, all the $10 \mathrm{kv}$ BATS had right operations and no power consumer lost their power supply. The $A B C$ system of YunPu substation was installed in 15th Oct 2014 and the ABC system of HeCun substation was installed in 16th Oct 2014. The $10 \mathrm{kv}$ IIA and IIB busbar load of YunPu substation and the $10 \mathrm{kv}$ IIA and IIB busbar load of HeCun substation were kept based on the real-time BATS control of the ABC system. The power supply reliability was assured greatly.

\section{Summarize}

This paper discusses the overall framework and functions of the $\mathrm{ABC}$ system. Some key issues such as its model, abnormal condition processing, testing technology and the time characters and so on are also provided. The application 
example of GuangZhou power grid shows that the BATS control policy is feasible and efficient. In the peak period of load, the BATS which will cause over load will be controlled to quit operation in certain logic. In the bottom period of load, if the operations of BATS don't bring over load, these BATS will be put into operation again to raise the reliability of power supply. This system is generally applicable and has great promotion value.

\section{References}

[1] Ding, S.W. (2007) Electric Power Systems Automechanism Theory. China Electric Power Press, Beijing.

[2] Li, X.Y., Wei, W., Wang, Y.H., et al.(2009) Study on the Development and Technology of Strong Smart Grid. Power System Protection and Control, 37, 1-6.

[3] Pan, K.Y., Liu, Z.Y., Song, X.Q., et al.(2009)Application of Automatic Load Controlling System in Foshan Power Grid. Automation of Electric Power Systems, 33, 9497.

[4] Li, S.Q., Zhang, M.J., Nin, X.H., et al.(2009) Automatic Loading Transfer Control Technology to Eliminate $330 \mathrm{kV}$ Transformer Overload. Power System Protection and Control), 37, 106-108.

[5] Pan, Q., Shi, B.N., Tang, J., et al.(2012) Study on Optimal Load Shedding Control to Eliminate Transformer Overload Based on Automatic Operation Mode Identification. Power System Protection and Control, 40, 125-128.

[6] Pan, K.Y. and Liu, Z.Y. (2013) Application of On_Lined Load-transfer Aided Decision System in Large Power Grid. Electrical Engineering, 5, 54-57.

[7] Wu, W.C., Sun, H.B., Zhang, B.M., et al. (2000) Simulation of Automatic Device Based on User-defined Model in Integrated EMS/DTS. Automation of Electric Power Systems, 24, 57-60.

[8] Pan, K.Y. and Tan, D.S. (2015) Optimal Strategy of on-line Strap Controlling of BATS Based on EMS. Electrical Engineering, 7, 104-107.

[9] Pan, K.Y., Tan, D.S., Zhao, Y., et al. (2015) A Fast Comutping Method of N-1 in Static Safety Analysis Considering BATS in Regional Power Grid, Automation of Electric Power Systems, 39, 122-126.

[10] Pan, K.Y., Tan, D.S., Li, J.G., et al. (2015) Optimal Strategy of Strap Controlling of BATS Considering Time-characteristics and Split-Characteristics. Electrical Engineering, 12, 102-105. 
Submit or recommend next manuscript to SCIRP and we will provide best service for you:

Accepting pre-submission inquiries through Email, Facebook, LinkedIn, Twitter, etc. A wide selection of journals (inclusive of 9 subjects, more than 200 journals)

Providing 24-hour high-quality service

User-friendly online submission system

Fair and swift peer-review system

Efficient typesetting and proofreading procedure

Display of the result of downloads and visits, as well as the number of cited articles Maximum dissemination of your research work

Submit your manuscript at: http://papersubmission.scirp.org/

Or contact epe@scirp.org 